\title{
1 Network Medicine Framework Shows Proximity of Polyphenol Targets and \\ 2 Disease Proteins is Predictive of the Therapeutic Effects of Polyphenols
}

4 Italo F. do Valle ${ }^{1}$, Harvey G. Roweth ${ }^{2,3}$, Michael W. Malloy ${ }^{2,3}$, Sofia Moco ${ }^{4}$, Denis

5 Barron ${ }^{4}$, Elisabeth Battinelli ${ }^{2,3}$, Joseph Loscalzo ${ }^{3,5}$, Albert-László Barabási ${ }^{1,6,7}$

${ }^{1}$ Network Science Institute and Department of Physics, Northeastern University, Boston, MA, USA

$8{ }^{2}$ Division of Hematology, Department of Medicine, Brigham and Women's Hospital, Boston, MA, USA

$9{ }^{3}$ Harvard Medical School, Boston, MA, USA

$10{ }^{4}$ Nestle Institute of Health Sciences, Lausanne, Switzerland

$11{ }^{5}$ Department of Medicine Brigham and Women's Hospital

$12{ }^{6}$ Channing Division of Network Medicine, Department of Medicine, Brigham and Women's Hospital, Harvard Medical

13 School, Boston, MA, USA

$14{ }^{7}$ Department of Network and Data Science, Central European University, Budapest, Hungary

\section{Abstract}

Polyphenols, natural products present in plant-based foods, play a protective role against several complex diseases through their antioxidant activity and by diverse

20 molecular mechanisms. Here we developed a network medicine framework to uncover

21 the mechanistic roles of polyphenols on health by considering the molecular interactions

22 between polyphenol protein targets and proteins associated with diseases. We find that

23 the protein targets of polyphenols cluster in specific neighborhoods of the human

24 interactome, whose network proximity to disease proteins is predictive of the molecule's

25 known therapeutic effects. The methodology recovers known associations, such as the

26 effect of epigallocatechin 3-O-gallate on type 2 diabetes, and predicts that rosmarinic

27 acid (RA) has a direct impact on platelet function, representing a novel mechanism

28 through which it could affect cardiovascular health. We experimentally confirm that RA

29 inhibits platelet aggregation and alpha granule secretion through inhibition of protein

30 tyrosine phosphorylation, offering direct support for the predicted molecular mechanism.

31 Our framework represents a starting point for mechanistic interpretation of the health

32 effects underlying food-related compounds, allowing us to integrate into a predictive

33 framework knowledge on food metabolism, bioavailability, and drug interaction. 


\section{Introduction}

36 Diet plays a defining role in human health. Indeed, while poor diet can significantly

37 increase the risk for coronary heart disease (CHD) and type 2 diabetes mellitus (T2D), a

38 healthy diet can play a protective role, even mitigating genetic risk for $\mathrm{CHD}^{1}$.

39 Polyphenols are a class of compounds present in plant-based foods, from fruits to vegetables, nuts, seeds, beans (e.g. coffee, cocoa), herbs, spices, tea, and wine, with well documented protective role as antioxidants, which affect several diseases, from cancer to T2D, cardiovascular, and neurodegenerative diseases ${ }^{2,3}$. Previous efforts profiled over 500 polyphenols in more than 400 foods $^{4,5}$ and have documented the high diversity of polyphenols to which humans are exposed through their diet, ranging from flavonoids to phenolic acids, lignans, and stilbenes.

The underlying molecular mechanisms through which specific polyphenols exert their beneficial effects on human health remain largely unexplored. From a mechanistic perspective, dietary polyphenols are not engaged in endogenous metabolic processes of anabolism and catabolism, but rather affect human health through their anti- or prooxidant activity ${ }^{6}$, by binding to proteins and modulating their activity ${ }^{7,8}$, interacting with digestive enzymes ${ }^{9}$, and modulating gut microbiota growth ${ }^{10,11}$. Yet, the variety of experimental settings and the limited scope of studies that explore the molecular effects of polyphenols have, to date, offered a range of often conflicting evidence. For example, two clinical trials, both limited in terms of the number of subjects and the intervention periods, resulted in conflicting conclusions about the beneficial effects of resveratrol on glycemic control in T2D patients ${ }^{12,13}$. We, therefore, need a framework to interpret the evidence present in the literature, and to offer in-depth mechanistic predictions on the molecular pathways responsible for the health implications of polyphenols present in diet. Ultimately, these insights could help us provide evidence on causal diet-health associations, guidelines of food consumption for different individuals, and help to

61 develop novel diagnostic and therapeutic strategies, which may lead to the synthesis of 62 novel drugs.

Here, we address this challenge by developing a network medicine framework to 
65 targets, unveiling their relationship to complex diseases. The developed framework is

66 based on the human interactome, a comprehensive subcellular network consisting of all

67 known physical interactions between human proteins, which has been validated

68 previously as a platform for understanding disease mechanisms ${ }^{14,15}$, rational drug target

69 identification, and drug repurposing ${ }^{16,17}$.

$70 \quad$ We find that the proteins to which polyphenols bind form identifiable

71 neighborhoods in the human interactome, allowing us to demonstrate that the proximity

72 between polyphenol targets and proteins associated with specific diseases is predictive

73 of the known therapeutic effects of polyphenols. Finally, we unveil the potential

74 therapeutic effects of rosmarinic acid (RA) on vascular diseases (V), predicting that its

75 mechanism of action is related to modulation of platelet function. We confirm this

76 prediction by experiments that indicate that RA modulates platelet function in vitro by

77 inhibiting tyrosine protein phosphorylation. Altogether, our results demonstrate that the

78 network-based relationship between disease proteins and polyphenol targets offers a

79 tool to systematically unveil the health effects of polyphenols.

81 Results

Polyphenol Targets Cluster in Specific Functional Neighborhoods of the Interactome

We mapped the targets of 65 polyphenols (see Methods) to the human interactome, consisting of 17,651 proteins and 351,393 interactions (Fig 1a,b). We find

87 that 19 of the 65 polyphenols have only one protein target, while a few polyphenols 88 have an exceptional number of targets (Fig 1c). We computed the Jaccard Index (JI) of

89 the protein targets of each polyphenol pair, finding only a limited similarity of targets

90 among different polyphenols (average $\mathrm{Jl}=0.0206$ ) (Supplementary Figure 1a). Even

91 though the average $\mathrm{Jl}$ is small, it is still significantly higher $(Z=147$, Supplementary

92 Figure $1 \mathrm{~b}$ ) than the $\mathrm{Jl}$ expected if the polyphenol targets were randomly assigned from

93 the pool of all network proteins with degrees matching the original set. This finding

94 suggests that while each polyphenol targets a specific set of proteins, their targets are

95 confined to a common pool of proteins, likely determined by commonalities in the 
96 polyphenol binding domains of the three-dimensional structure of the protein targets ${ }^{18}$.

97 Gene Ontology (GO) Enrichment Analysis recovers existing mechanisms ${ }^{8}$ and also

98 helps identify new processes related to polyphenol protein targets, such as post-

99 translation protein modifications, regulation, and xenobiotic metabolism (Fig 1d). The

100 enriched GO categories indicate that polyphenols modulate common regulatory

101 processes, but the low similarity in their protein targets, illustrated by the low average Jl,

102 indicates that they target different processes within the same process.

103 We next asked whether the polyphenol targets cluster in specific regions of the

104 human interactome. We focused on polyphenols with more than two targets ( $\mathrm{n}=46$, Fig

105 2), and measured the size and significance of the largest connected component (LCC)

106 formed by the targets of each polyphenol. We found that 25 of the 46 polyphenols have

107 a larger LCC than expected by chance (Z-score > 1.95) (Fig 1e, Fig 2). In agreement

108 with experimental evidence documenting the effect of polyphenols on multiple

109 pathways $^{19}$, we find that ten polyphenols have their targets organized in multiple

110 connected components of size $>2$.

111 These results indicate that the targets of polyphenols modulate specific well

112 localized neighborhoods of the interactome (Fig 2, Supplementary Figure 1c). This

113 prompted us to explore if the interactome regions targeted by the polyphenols reside

114 within network neighborhoods associated with specific diseases, seeking a network-

115 based framework to unveil the molecular mechanism through which specific

116 polyphenols modulate health.

118 Proximity Between Polyphenol Targets and Disease Proteins Reveals their Therapeutic

119 Effects

121 Polyphenols can be viewed as drugs in that they bind to specific proteins, affecting their

122 ability to perform their normal functions. We, therefore, hypothesized that we can apply

123 the network-based framework used to predict the efficacy of drugs in specific

124 diseases $^{16,17}$ to also predict the therapeutic effects of polyphenols. The closer the

125 targets of a polyphenol are to disease proteins, the more likely that the polyphenol will

126 affect the disease phenotype. We, therefore, calculated the network proximity between 
127 polyphenol targets and proteins associated with 299 diseases using the closest

128 measure, $d_{c}$, representing the average shortest path length between each polyphenol

129 target and the nearest disease protein (see Methods). Consider for example (-)-

130 epigallocatechin 3-O-gallate (EGCG), a polyphenol abundant in green tea.

131 Epidemiological studies have found a positive relationship between green tea

132 consumption and reduced risk of $\mathrm{T}^{2} \mathrm{D}^{20,21}$, and physiological and biochemical studies

133 have shown that EGCG presents glucose-lowering effects in both in vitro and in vivo

134 models $^{22,23}$. We identified 54 experimentally validated EGCG protein targets and

135 mapped them to the interactome, finding that the ECGC targets form an LCC of 17

136 proteins $(Z=7.61)$ (Fig 3a). We also computed the network-based distance between

137 EGCG targets and 83 proteins associated with T2D, finding that the two sets are

138 significantly proximal to each other. We ranked all 299 diseases based on the network

139 proximity to the ECGC targets in order to determine whether we can recover the 82

140 diseases in which ECGC has known therapeutic effects according to the CTD database.

141 By this analysis, we were able to recover 15 previously known therapeutic associations

142 among the top 20 ranked diseases (Table 1), confirming that network-proximity can

143 discriminate between known and unknown disease associations for polyphenols, as

144 previously confirmed among drugs ${ }^{16,17}$.

145 We expanded these methods to all polyphenol-disease pairs, to predict diseases

146 for which specific polyphenols might have therapeutic effects. For this analysis, we

147 grouped all 19,435 polyphenol-disease associations between 65 polyphenols and 299

148 diseases into known $(1,525)$ and unknown $(17,910)$ associations. The known

149 polyphenol-disease set was retrieved from CTD, which is limited to manually curated

150 associations for which there is literature-based evidence. For each polyphenol, we

151 tested how well network proximity discriminates between the known and unknown sets

152 by evaluating the area under the Receiving Operating Characteristic (ROC) curve

153 (AUC). For EGCG, network proximity offers good discriminative power $(\mathrm{AUC}=0.78, \mathrm{Cl}$ :

1540.70 - 0.86) between diseases with known and unknown therapeutic associations (Table

155 1). We find that network proximity $\left(d_{c}\right)$ offers predictive power with an AUC > 0.7 for 31

156 polyphenols (Fig 3b). The methodology recovers many associations well documented in

157 the literature, like the beneficial effects of umbelliferone on colonrectal neoplasms ${ }^{24,25}$. 
158 In Table 2 we summarize the top 10 polyphenols for which the network medicine

159 framework offers the best predictive power of therapeutic effects, limiting the entries to

160 predictive performance of $A \cup C>0.6$ and performance over top predictions with

161 precision > 0.6. Given the lack of data on true negative examples, we considered

162 unknown associations as negative cases, observing the same trend when we used an

163 alternative performance metric that does not require true negative labels (i.e. AUC of

164 the Precision-Recall curve) (Supplementary Figure 2).

165 Finally, we performed multiple robustness checks to exclude the role of potential

166 biases in the input data. To test if the predictions are biased by the set of known

167 associations retrieved from CTD, we randomly selected 100 papers from PubMed

168 containing MeSH terms that tag EGCG to diseases. We manually curated the evidence

169 for EGCG's therapeutic effects for the diseases discussed in the published papers,

170 excluding reviews and non-English language publications. The dataset was processed

171 to include implicit associations (see Methods), resulting in a total of 113 diseases

172 associated with EGCG, of which 58 overlap with the associations reported by CTD (Fig

173 3c). We observed that the predictive power of network proximity was unaffected by

174 whether we considered the annotations from CTD, the manually curated list, or the

175 union of both (Fig 3d). To test the role of potential biases in the interactome, we

176 repeated our analysis using only high-quality polyphenol-protein interactions retrieved

177 from ligand-protein 3D resolved structures (Supplementary Figure 1d) and a subset of

178 the interactome derived from an unbiased high-throughput screening (Supplementary

179 Figure 1f). We find that the predictive power was largely unchanged, indicating that the

180 literature bias in the interactome does not affect our findings. Finally, we re-tested the

181 predictive performance by considering not only the therapeutic polyphenol-disease

182 associations, but also the marker/mechanism ones - another type of curated association

183 available in CTD - finding that the predictive power remains largely unchanged

184 (Supplementary Notes, Supplementary Figure 3).

186 Network Proximity Predicts Gene Expression Perturbation Induced by Polyphenols 
188 To validate that network proximity reflects biological activity of polyphenols observed in

189 experimental data, we retrieved expression perturbation signatures from the

190 Connectivity Map database ${ }^{26}$ for the treatment of the breast cancer MCF7 cell line with

19121 polyphenols (Supplementary Table 1, Supplementary Figure 4). We investigated the

192 relationship between the extent to which polyphenols perturb the expression of disease

193 genes, the network proximity between the polyphenol targets and disease proteins, and

194 their known therapeutic effects (Fig 4a). For example, we observe different perturbation

195 profiles for gene pools associated with different diseases: for treatment with genistein (1

$196 \mu \mathrm{M}, 6$ hours) we observe 10 skin disease genes with perturbation score $>2$, while we

197 observe only one highly perturbed cerebrovascular disorder gene (Fig 4b). Indeed,

198 network proximity indicates that skin disease is closer to the genistein targets than

199 cerebrovascular disorder, suggesting a relationship between network proximity, gene

200 expression perturbation, and the therapeutic effects of the polyphenol (Fig 4a). To test

201 this hypothesis, we computed an enrichment score that measures the

202 overrepresentation of disease genes among the most perturbed genes (see Methods),

203 finding 13 diseases that have their genes significantly enriched among the most

204 deregulated genes by genistein, of which 4 have known therapeutic associations. We

205 find that these four diseases are significantly closer to the genistein targets than the

206 nine diseases with unknown therapeutic associations (Fig 4c). We observed a similar

207 trend for treatments with other polyphenols, whether we use the same $(1 \mu \mathrm{M}, \mathrm{Fig} 4 \mathrm{c})$ or

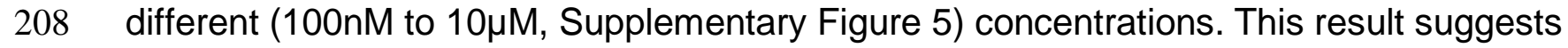

209 that changes in gene expression caused by a polyphenol are indicative of its therapeutic

210 effects, but only if the observed expression change is limited to proteins proximal to the

211 polyphenol targets (Fig 4a).

212 Consequently, network proximity should also be predictive of the overall gene

213 expression perturbation caused by a polyphenol on the genes of a given disease. To

214 test this hypothesis, in each experimental combination defined by the polyphenol type

215 and its concentration, we evaluated the maximum perturbation among genes for each

216 disease. We then compared the magnitude of the observed perturbation between

217 diseases that were proximal $\left(d_{c}<25^{\text {th }}\right.$ percentile, $\left.Z_{d_{c}}<-0.5\right)$ or distal $\left(d_{c}>75^{\text {th }}\right.$

218 percentile, $\left.Z_{d_{c}}>-0.5\right)$ to the polyphenol targets. Figures 5a-b and Supplementary Figure 
2196 show the results for the genistein treatment ( $1 \mu \mathrm{M}, 6$ hours), indicating that diseases

220 proximal to the polyphenol targets show higher maximum perturbation values than distal

221 diseases. The same trend is observed for other polyphenols when we use different $d_{c}$

222 and $Z_{d_{c}}$ thresholds for defining proximal and distant diseases (Figs $5 \mathrm{~b}$, Supplementary

223 Figures 6-9), confirming that the impact of a polyphenol on cellular signaling pathways

224 is localized in the network space, being greater in the vicinity of the polyphenol targets

225 compared to neighborhoods remote from these targets. We also considered gene

226 expression perturbations in the network vicinity of the polyphenol targets, regardless of

227 whether the proteins were disease proteins or not, observing higher perturbation scores

228 for proximal proteins in 12 out 21 polyphenols tested at $10 \mu \mathrm{M}$ (Supplementary Figure

229 10). Finally, we find that the enrichment score of perturbed genes among disease genes

230 is not as predictive of the polyphenol therapeutic effects as network proximity

231 (Supplementary Figure 11).

$232 \quad$ Altogether these results indicate that network proximity offers a mechanistic

233 interpretation for the gene expression perturbations induced by polyphenols on disease

234 genes. They also show that network proximity can indicate when gene expression

235 perturbations result in therapeutic effects, suggesting that future studies could integrate

236 gene expression (whenever available) with network proximity as they aim to more

237 accurately prioritize polyphenol-disease associations.

Experimental Evidence Confirms that Rosmarinic Acid Modulates Platelet Function

To demonstrate how the network-based framework can facilitate the mechanistic

242 interpretation of the therapeutic effects of selected polyphenols, we next focus on

243 vascular diseases (V). Of 65 polyphenols evaluated in this study, we found 27 to have

244 associations to $\mathrm{V}$, as their targets were within the $\mathrm{V}$ network neighborhood

245 (Supplementary Table 3). We, therefore, inspected the targets of 15 of the 27

246 polyphenols with 10 or less targets. The network analysis identified direct links between

247 biological processes related to vascular health and the targets of three polyphenols:

248 gallic acid, rosmarinic acid, and 1,4-naphthoquinone (Supplementary Figure 12,

249 Supplementary Notes). The network neighborhood containing the targets of these 
250 polyphenols suggests that gallic acid activity involves thrombus dissolution processes,

251 rosmarinic acid acts on platelet activation and antioxidant pathways through FYN and its

252 neighbors, and 1,4-naphthoquinone acts on signaling pathways of vascular cells

253 through MAP2K1 activity (Supplementary Figure 12, Supplementary Notes).

To validate the developed framework, we set out to obtain direct experimental evidence of the predicted mechanistic role of rosmarinic acid (RA) in V. The RA targets

256 are in close proximity to proteins related to platelet function, forming the RA-V-platelet

257 module: a connected component formed by the RA target FYN and the $\mathrm{V}$ proteins

258 associated with platelet function PDE4D, CD36, and APP (Fig 6a). We, therefore, asked

259 whether RA influenced platelet activation in vitro. As platelets can be stimulated through

260 different activation pathways, RA effects can, in principle, occur in any of them. To test

261 these different possibilities, we pretreated platelets with RA and then activated with: 1)

262 glycoprotein VI by collagen or collagen-related peptide (CRP/CRPXL); 2) protease-

263 activated receptors-1,4 by thrombin receptor activator peptide-6 (TRAP-6); 3)

264 prostanoid thromboxane receptor by the thromboxane $A_{2}$ analogue (U46619); and 4)

$265 \mathrm{P} 2 \mathrm{Y} 1 / 12$ receptor by adenosine diphosphate (ADP) ${ }^{27}$. When we compared the network

266 distance between each stimulant receptor and the RA-V-platelet module (Fig 6a), we

267 observed that the receptors for CRP/CRPXL, TRAP-6, and U46619 are closer than

268 random expectation, while the receptor for ADP is more distant (Fig 6b). We expected

269 that platelets would be most affected by RA when treated with stimulants whose

270 receptors are most proximal to the RA-V-platelet module, i.e., CRP/CRPXL, TRAP-6,

271 and U46619, and as a control, we expect no effect for the distant ADP receptor. The

272 experiments confirm this prediction: RA inhibits collagen-mediated platelet aggregation

273 (Fig 6c) and impairs dense granule secretion induced by CRPXL, TRAP-6, and U46619

274 (Supplementary Figure 13). RA-treated platelets also displayed dampened alpha-

275 granule secretion (Fig 6d) and integrin $\alpha$ llbß33 activation (Supplementary Figure 13) in

276 response to U46619. As expected, RA did not affect platelet function when we used an

277 agonist whose receptor is distant from the RA-V-module, i.e., ADP. These findings

278 suggest that RA impairs basic hallmarks of platelet activation via strong network effects,

279 supporting our hypothesis that the proximity between RA targets and the neighborhood

280 associated with platelet function (Fig 6a) could in part explain RA's impact on V. 
We next searched to clarify the molecular mechanisms involved in the impact of

282 RA on platelets. Given that platelet activation is coordinated by several kinases, we

283 hypothesized that RA inhibits platelet function by blocking agonist-induced protein

284 tyrosine phosphorylation. We observed that RA-treated platelets demonstrated a dose-

285 dependent reduction in total tyrosine phosphorylation in response to CRPXL, TRAP-6

286 and U46619 (Fig 6e). Given that RA caused a substantial decrease in phosphorylation

287 of proteins with atomic mass between 50-60 KDa (Fig 6e), we hypothesized that RA

288 may reduce phosphorylation of FYN (59 KDa), or other similarly sized members of the

289 same protein family (i.e. src family kinases, SFKs). To test this, we measured the level

290 of phosphorylation within the activation domain (amino acid 416) of SFKs, finding that

291 RA reduced collagen induced phosphorylation of FYN as well as basal tyrosine

292 phosphorylation of SFKs (Fig 6f). This indicates that RA perturbs the phospho-signaling

293 networks that regulate platelet response to extracellular stimuli.

294 Altogether, these findings support our prediction that RA modulates platelet

295 activation and function. It also supports the observation that its mechanism of action

296 involves reduction of phosphorylation at the activation domain of the protein-tyrosine

297 kinase FYN (Fig 6a) and the inhibition of general tyrosine phosphorylation. Finally, while

298 polyphenols are usually associated to their antioxidant function, here we illustrate

299 another mechanistic pathway through which they could benefit health.

\section{Discussion}

303 Here, we proposed a network-based framework to predict the therapeutic effects of

304 dietary polyphenols in human diseases. We find that polyphenol protein targets cluster

305 in specific functional neighborhoods of the interactome, and we show that the network

306 proximity between polyphenol targets and disease proteins is predictive of the

307 therapeutic effects of polyphenols. We demonstrate that diseases whose proteins are

308 proximal to polyphenol targets tend to have significant changes in gene expression in

309 cell lines treated with the respective polyphenol, while such changes are absent for

310 diseases whose proteins are distal to polyphenol targets. Finally, we find that the

311 network neighborhood around the RA targets and vascular disease proteins are related 
312 to platelet function. We validate this mechanistic prediction by showing that RA

313 modulates platelet function through inhibition of protein tyrosine phosphorylation. These

314 observations suggest a role of RA on prevention of vascular diseases by inhibiting

315 platelet activation and aggregation.

316 The observed results also suggest multiple avenues through which our ability to

317 understand the role of polyphenols could be improved. First, some of the known health

318 benefits of polyphenols might be caused not only by the native molecules, but also by

319 their metabolic byproducts ${ }^{28,29}$. We, however, lack data about colonic degradation, liver

320 metabolism, bioavailability, and interaction with proteins of specific polyphenols or their

321 metabolic byproducts. Future experimental data on protein interactions with polyphenol

322 byproducts and conjugates can be incorporated in the proposed framework, further

323 improving the accuracy of our predictions. The lack of this data does not invalidate the

324 findings presented here, since previous studies report the presence of unmetabolized

325 polyphenols in blood ${ }^{30-32}$; and it has been hypothesized that, in some instances,

326 deconjugation of liver metabolites occurs in specific tissues or cells ${ }^{33-35}$. Therefore, the

327 lack of data for specific polyphenols and the fact that other mechanisms exist through

328 which they can affect health (e.g. antioxidant activity, microbiota regulation) explain why

329 this methodology might still miss a few known relationships between polyphenols and

330 diseases. Second, considering that several experimental studies of polyphenol

331 bioefficacy have been observed in in vitro and in vivo models, the proposed framework

332 might help us interpret literature evidence, possibly even allowing us to exclude

333 chemical candidates when considering the health benefits provided by a given food in

334 epidemiological association studies.

335 Our assumption that network proximity recovers therapeutic associations is

336 based on its predictive performance on a ground truth dataset for observed therapeutic

337 effects and also relies on previous observations about the effect of drugs on

338 diseases ${ }^{16,17,36}$. While the proposed methodology offers a powerful prioritization tool to

339 guide future research, the real effect of polyphenols on diseases might still be negative,

340 given other unmet factors such as dosage, comorbidities, and drug interactions, which

341 can only be ruled out by pre-clinical and clinical studies. Gene expression perturbation

342 profiles, such as the ones provided by the Connectivity map, can also be integrated with 
343 network proximity to further highlight potential beneficial or harmful effects of chemical 344 compounds ${ }^{37,38}$.

345 The low bioavailability of some polyphenols in food might still present challenges 346 when considering the therapeutic utility of these molecules. However, 48 of the 65

347 polyphenols we explored here are predicted to have high gastrointestinal absorption

348 (Supplementary Table 2) and different methodologies are available to increase

349 bioavailability of natural compounds ${ }^{39,40}$. Additionally, in the same way that the

350 polyphenol phlorizin led to the discovery of new strategies for disease treatment

351 resulting in the development of new compounds with higher efficacy ${ }^{41}$, we believe that

352 the present methodology can help us identify polyphenol-based candidates for drug 353 development.

$354 \quad$ The methodology introduced here offers a foundation for the mechanistic

355 interpretation of alternative pathways through which polyphenols can affect health, e.g., 356 the combined effect of different polyphenols ${ }^{36,42}$ and their interaction with drugs ${ }^{43}$. To

357 address such synergistic effects, we need ground-truth data on these aspects. The 358 developed methodology can be applied to other food-related chemicals, providing a 359 framework by which to understand their health effects. Future research may help us 360 also account for the way that food-related chemicals affect endogenous metabolic 361 reactions, impacting not only signaling pathways, but also catabolic and anabolic 362 processes. Finally, the methodology provides a framework to interpret and find causal 363 support for associations identified in observational studies. Taken together, the 364 proposed network-based framework has the potential to reveal systematically the 365 mechanism of action underlying the health benefits of polyphenols, offering a logical, 366 rational strategy for mechanism-based drug development of food-based compounds.

\section{Methods}

\section{Building the Interactome}

372 The human interactome was assembled from 16 databases containing different types of 373 protein-protein interactions (PPIs): 1) binary PPIs tested by high-throughput yeast-two- 
374 hybrid (Y2H) experiments ${ }^{44}$; 2) kinase-substrate interactions from literature-derived low-

375 throughput and high-throughput experiments from KinomeNetwork ${ }^{45}$, Human Protein

376 Resource Database (HPRD) ${ }^{46}$, and PhosphositePlus ${ }^{47}$; 3) carefully literature-curated

377 PPIs identified by affinity purification followed by mass spectrometry (AP-MS), and from

378 literature-derived low-throughput experiments from InWeb ${ }^{48}$, BioGRID ${ }^{49}, \mathrm{PINA}^{50}$,

$379 \mathrm{HPRD}^{51}, \mathrm{MINT}^{52}$, IntAct ${ }^{52}$, and InnateDB ${ }^{53}$; 4) high-quality PPIs from three-dimensional

380 (3D) protein structures reported in Instruct ${ }^{54}$, Interactome3D ${ }^{55}$, and INSIDER ${ }^{56}$; 5)

381 signaling networks from literature-derived low-throughput experiments as annotated in

382 SignaLink2.0 ${ }^{57}$; and 6) protein complex from BioPlex2.0 ${ }^{58}$. The genes were mapped to

383 their Entrez ID based on the National Center for Biotechnology Information (NCBI)

384 database as well as their official gene symbols. The resulting interactome includes

385351,444 protein-protein interactions (PPIs) connecting 17,706 unique proteins

386 (Supplementary Data 1). The largest connected component has 351,393 PPIs and

38717,651 proteins.

Polyphenols, Polyphenol Targets, and Disease Proteins

We retrieved 759 polyphenols from the PhenolExplorer database ${ }^{4}$. The database lists

392 polyphenols with food composition data or profiled in biofluids after interventions with

393 polyphenol-rich diets. For our analysis, we only considered polyphenols that: 1) could

394 be mapped in PubChem IDs, 2) were listed in the Comparative Toxicogenomics (CTD)

395 database ${ }^{59}$ as having therapeutic effects on human diseases, and 3) had protein-

396 binding information present in the STITCH database ${ }^{60}$ with experimental evidence (Fig

397 1a). After these steps, we considered a final list of 65 polyphenols, for which 598 protein

398 targets were retrieved from STITCH (Supplementary Table 1). We considered 3,173

399 disease proteins corresponding to 299 diseases retrieved from Menche et al (2015) ${ }^{15}$.

400 Gene ontology enrichment analysis of protein targets was performed using the

401 Bioconductor package clusterProfiler with a significance threshold of $p<0.05$ and

402 Benjamini-Hochberg multiple testing correction with $q<0.05$. 
We retrieved the polyphenol-disease associations from the Comparative

407 Toxicogenomics Database (CTD). We considered only manually curated associations

408 labeled as therapeutic. By considering the hierarchical structure of diseases along the

409 MeSH tree, we expanded explicit polyphenol-disease associations to include also

410 implicit associations. This procedure was performed by propagating associations in the

411 lower branches of the MeSH tree to consider diseases in the higher levels of the same

412 tree branch. For example, a polyphenol associated with heart diseases would also be

413 associated with the more general category of cardiovascular diseases. By performing

414 this expansion, we obtained a final list of 1,525 known associations between the 65

415 polyphenols and the 299 diseases considered in this study.

Network Proximity Between Polyphenol Targets and Disease Proteins

The proximity between a disease and a polyphenol was evaluated using a distance metric that takes into account the shortest path lengths between polyphenol targets and disease proteins ${ }^{16}$. Given $S$, the set of disease proteins, $T$, the set of polyphenol targets, and $d(s, t)$, the shortest path length between nodes $s$ and $t$ in the network, we define:

$$
d_{c}(S, T)=\frac{1}{\|T\|} \sum_{t \in T} \min _{s \in S} d(s, t)
$$

425 We also calculated a relative distance metric $\left(Z_{d c}\right)$ that compares the absolute distance $426 d_{c}(S, T)$ between a disease and a polyphenol with a reference distribution describing the 427 random expectation. The reference distribution corresponds to the expected distances 428 between two randomly selected groups of proteins matching the size and degrees of the 429 original disease proteins and polyphenol targets in the network. It was generated by 430 calculating the proximity between these two randomly selected groups across 1,000 431 iterations. The mean $\mu_{d(S, T)}$ and standard deviation $\sigma_{d(S, T)}$ of the reference distribution 432 were used to convert the absolute distance $d_{c}$ into the relative distance $Z_{d c}$, defined as:

$$
Z_{d_{c}}=\frac{d-\mu_{d_{c}(S, T)}}{\sigma_{d_{c}(S, T)}}
$$


436 We performed a degree-preserving random selection, but due to the scale-free nature

437 of the human interactome, we avoid repeatedly choosing the same (high degree) nodes

438 by using a binning approach in which nodes within a certain degree interval were

439 grouped together such that there were at least 100 nodes in the bin. The

440 Supplementary Data 2 reports the proximity scores $d_{c}$ and $Z_{d_{c}}$ for all pairs of diseases

441 and polyphenols.

443 Area Under ROC Curve Analysis

445 For each polyphenol, we used AUC to evaluate how well the network proximity

446 distinguishes diseases with known therapeutic associations from all of the others of the

447 set of 299 diseases. The set of known associations (therapeutic) retrieved from CTD

448 were used as positive instances, all unknown associations were defined as negative

449 instances, and the area under the ROC curve was computed using the implementation

450 in the Scikit-learn Python package. Furthermore, we calculated 95\% confidence

451 intervals using the bootstrap technique with 2,000 resamplings with sample sizes of 150

452 each. Considering that AUC provides an overall performance, we also searched for a

453 metric to evaluate the top ranking predictions. For this analysis, we calculated the

454 precision of the top 10 predictions, considering only the polyphenol-disease

455 associations with relative distance $Z_{d c}<-0.5^{16}$.

457 Analysis of Network Proximity and Gene Expression Deregulation

459 We retrieved perturbation signatures from the Connectivity Map database

460 (https://clue.io/) for the MCF7 cell line after treatment with 21 polyphenols. These

461 signatures reflect the perturbation of the gene expression profile caused by the

462 treatment with that particular polyphenol relative to a reference population, which

463 comprises all other treatments in the same experimental plate ${ }^{26}$. For polyphenols having

464 more than one experimental instance (time of exposure, cell line, dose), we selected the 465 one with highest distil_cc_q75 value (75th quantile of pairwise spearman correlations in 
466 landmark genes, https://clue.io/connectopedia/perturbagen\types\_and \_controls). We

467 performed Gene Set Enrichment Analysis ${ }^{61}$ to evaluate the enrichment of disease

468 genes among the top deregulated genes in the perturbation profiles. This analysis offers

469 Enrichment Scores (ES) that have small values when genes are randomly distributed

470 among the ordered list of expression values and high values when they are

471 concentrated at the top or bottom of the list. The ES significance is calculated by

472 creating 1,000 random selection of gene sets with the same size as the original set and

473 calculating an empirical p-value by considering the proportion of random sets resulting

474 in ES smaller than the original case. The $\mathrm{p}$-values were adjusted for multiple testing

475 using the Benjamini-Hochberg method. The network proximity $d_{c}$ of disease proteins

476 and polyphenol targets for diseases with significant ES were compared according to

477 their therapeutic and unknown-therapeutic associations using the Student's t-test. The

478 relevant code for calculating the network proximity, AUCs, and enrichment scores can

479 be found on https://github.com/italodovalle/polyphenols.

$\underline{\text { Platelet Isolation }}$

483 Human blood collection was performed as previously described in accordance with the 484 Declaration of Helsinki and ethics regulations with Institutional Review Board approval 485 from Brigham and Women's Hospital (P001526). Healthy volunteers did not ingest 486 known platelet inhibitors for at least 10 days prior. Citrated whole blood underwent 487 centrifugation with a slow brake (177 x g, 20 minutes), and the PRP fraction was 488 acquired for subsequent experiments. For washed platelets, PRP was incubated with 1 $489 \mu \mathrm{M}$ prostaglandin $\mathrm{E}_{1}$ (Sigma, P5515) and immediately underwent centrifugation with a 490 slow brake (1000 x g, 5 minutes). Platelet-poor plasma was aspirated, and pellets 491 resuspended in platelet resuspension buffer (PRB; 10 mM Hepes, 140 mM NaCl, 3 mM $492 \mathrm{KCl}, 0.5 \mathrm{mM} \mathrm{MgCl}_{2}, 5 \mathrm{mM} \mathrm{NaHCO}_{3}, 10 \mathrm{mM}$ glucose, $\mathrm{pH}$ 7.4).

\section{$494 \quad$ Platelet Aggregometry}


496 Platelet aggregation was measured by turbidimetric aggregometry as previously

497 described $^{62}$. Briefly, PRP was pretreated with RA for 1 hour before adding $250 \mu \mathrm{L}$ to

498 siliconized glass cuvettes containing magnetic stir bars. Samples were placed in

499 Chrono-Log ${ }^{\circledR}$ Model 700 Aggregometers before the addition of various platelet agonists.

500 Platelet aggregation was monitored for 6 minutes at $37^{\circ} \mathrm{C}$ with a stir speed of $1000 \mathrm{rpm}$

501 and the maximum extend of aggregation recorded using AGGRO/LINK ${ }^{\circledR} 8$ software. In

502 some cases, dense granule release was simultaneously recorded by supplementing

503 samples with Chrono-Lume ${ }^{\circledR}\left(\right.$ Chrono-Log $\left.^{\circledR}, 395\right)$ according to the manufacturer's

504 instructions.

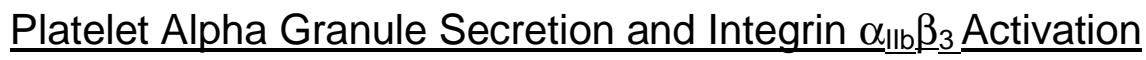

508 Changes in platelet surface expression of P-selectin (CD62P) or binding of Alexa

509 Fluor $^{\mathrm{TM}}$ 488-conjugated fibrinogen were used to assess alpha granule secretion and

510 integrin $\alpha_{11 b} \beta_{3}$ activation, respectively. First, PRP was pre-incubated with RA for 1 hour,

511 followed by stimulation with various platelet agonists under static conditions at $37^{\circ} \mathrm{C}$ for

51220 minutes. Samples were then incubated with APC-conjugated anti-human CD62P

513 antibodies (BioLegend ${ }^{\circledR}, 304910$ ) and $100 \mu \mathrm{g} / \mathrm{mL}$ Alexa Fluor ${ }^{\mathrm{TM}}$ 488-Fibrinogen (Thermo

514 Scientific $^{\mathrm{TM}}, \mathrm{F} 13191$ ) for 20 minutes before fixation in $2 \%$ [v/v] paraformaldehyde

515 (Thermo Scientific ${ }^{\text {TM }}$, AAJ19945K2). Fifty thousand platelets were processed per

516 sample using a Cytek ${ }^{\mathrm{TM}}$ Aurora spectral flow cytometer. Percent-positive cells were

517 determined by gating on fluorescence intensity compared to unstimulated samples.

Platelet Cytotoxicity

521 Cytotoxicity were tested by measuring lactate dehydrogenase (LDH) release by

522 permeabilized platelets into the supernatant ${ }^{63}$. Briefly, washed platelets were treated

523 with various concentrations of RA for 1 hour, before isolating supernatants via

524 centrifugation (15,000 x g, 5 min). A Pierce LDH Activity Kit (Thermo Scientific ${ }^{\text {TM }}$,

525 88953) was then used to assess supernatant levels of LDH. 
Washed platelets were pre-treated with RA for 1 hour, followed by a 15 minute

530 treatment with Eptifibatide $(50 \mu \mathrm{M})$. Platelets were then stimulated with various agonists

531 for 5 minutes under stirring conditions (1000 rpm, $\left.37^{\circ} \mathrm{C}\right)$. Platelets were lysed on ice

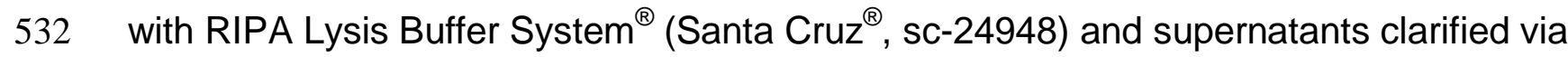

533 centrifugation $\left(15,000 \times \mathrm{g}, 10 \mathrm{~min}, 4^{\circ} \mathrm{C}\right)$. For immunoprecipitation of $\mathrm{FYN}$, lysates were

534 first precleared of IgG by incubating with Protein A agarose beads (Cell Signaling

535 Technologies, 9863S) for 30 minutes at $4^{\circ} \mathrm{C}$, before isolation of the supernatant via

536 centrifugation $\left(15,000 \times \mathrm{g}, 10 \mathrm{~min}, 4^{\circ} \mathrm{C}\right)$. Supernatants were incubated with anti-FYN

537 antibodies (Abcam, 2A10) overnight at $4^{\circ} \mathrm{C}$ before incubation with Protein $\mathrm{A}$ beads for 1

538 hour. Beads were then washed 5 times with NP-40 lysis buffer (144 mM Tris, 518 mM

$539 \mathrm{NaCl}, 6 \mathrm{mM}$ EDTA, $12 \mathrm{mM} \mathrm{Na}_{2} \mathrm{VO}_{3}, 33.3 \%$ [v/v] NP-40, Halt ${ }^{\mathrm{TM}}$ protease inhibitor

540 cocktail (Thermo, 78429)).

$541 \quad$ For Western Blot analysis, total cell lysates or immunoprecipitated FYN were

542 reduced with Laemmli Sample Buffer (Bio-Rad, 1610737) and proteins separated by

543 molecular weight in PROTEAN TGX ${ }^{\mathrm{TM}}$ precast gels (Bio-Rad, 4561084). Proteins were

544 transferred to PVDF membranes (Bio-Rad, 1620174) and probed with either 4G10

545 (Milipore, 05-321), a primary antibody clone that recognizes phosphorylated tyrosine

546 residues, or primary antibodies that probe for the site-specific phosphorylation of src

547 family kinases (SFKs, p-Tyr416) within their activation loop. Membranes were incubated

548 with horseradish peroxidase-conjugated secondary antibodies (Cell Signaling

549 Technologies, 7074S) to catalyze an electrochemiluminescent reaction (Thermo

550 Scientific $\left.^{\mathrm{TM}}, \mathrm{PI} 32109\right)$. Membranes were visualized using a Bio-Rad ChemiDoc Imaging

551 System and densitometric analysis of protein lanes conducted using ImageJ $(\mathrm{NIH}$,

552 Version 1.52a).

\section{Author Contributions}

556 I.F.V and A.L.B designed the study. I.F.V. performed all computational analyses. H.G.R,

557 M.W.M., E.B., and J.L designed and performed experimental validation. J.L. guided 
558 I.F.V. on validation case studies. S.M and D.B guided I.F.V for data interpretation and

559 curation of disease associations obtained from literature. I.F.V and A.L.B wrote the

560 paper with input from all authors. All authors read and approved the manuscript.

Acknowledgements

564 This study was supported, in part, by NIH grant 1P01HL132825, HG007690, HL108630, 565 and HL119145; American Heart Association grants 151708 and D700382; and ERC

566 grant 810115-DYNASET. We would like to thank Peter Ruppert, Giulia Menichetti, and

567 Istvan Kovacs for support in this study, Feixiong Cheng for assembling the Human

568 Interactome, and Alice Grishchenko for help with data visualization.

\section{Declaration of Interests}

572 J.L. and A.L.B are co-scientific founder of Scipher Medicine, Inc., which applies network 573 medicine strategies to biomarker development and personalized drug selection. A.L.B is

574 the founder of Nomix Inc. and Foodome, Inc. that apply data science to health; I.F.V is a 575 scientific consultant for Foodome, Inc.

\section{Data Availability}

579 The authors declare that all data supporting the findings of this study are available at

580 https://github.com/italodovalle/polyphenols and within the paper and its supplementary 581 information files.

583 Code Availability

585 Computer code is available at https://github.com/italodovalle/polyphenols 
588 Table 1 - Top 20 Predicted Therapeutic Associations Between EGCG and Human

589 Diseases. Diseases were ordered according to the network distance $\left(d_{c}\right)$ of their

590 proteins to EGCG targets and diseases with relative distance $Z_{d c}>-0.5$ were removed.

591 References reported in CTD for curated 'therapeutic associations' are shown.

592

\begin{tabular}{|c|c|c|c|}
\hline Disease & Distance $d_{c}$ & Significance $Z_{d_{c}}$ & $\begin{array}{c}\text { Known Therapeutic Effect } \\
\text { (References) }\end{array}$ \\
\hline nervous system diseases & 1.13 & -1.72 & 64,65 \\
\hline metabolic diseases & 1.25 & -1.41 & 23 \\
\hline cardiovascular diseases & 1.27 & -2.67 & $66-71$ \\
\hline vascular diseases & 1.33 & -3.47 & $66,67,70$ \\
\hline digestive system diseases & 1.33 & -1.57 & $73-77$ \\
\hline neurodegenerative diseases & 1.37 & -1.71 & 78 \\
\hline central nervous system diseases & 1.41 & -0.54 & 78 \\
\hline brain diseases & 1.43 & -0.89 & NA \\
\hline intestinal diseases & 1.49 & -1.08 & 79 \\
\hline inflammatory bowel diseases & 1.54 & -2.10 & $\mathrm{NA}$ \\
\hline bone diseases & 1.54 & -1.18 & $\mathrm{NA}$ \\
\hline gastroenteritis & 1.54 & -1.92 & NA \\
\hline demyelinating diseases & 1.54 & -1.78 & NA \\
\hline glucose metabolism disorders & 1.54 & -1.58 & 23 \\
\hline heart diseases & 1.56 & -1.20 & $68,69,71$ \\
\hline
\end{tabular}

593 
594 Table 2 - Top Ranked Polyphenols. Polyphenols for which network proximity to 595 diseases best predicts their therapeutic effects. Table showing polyphenols with AUC > 5960.6 and Precision $>0.6$. $\left({ }^{*}\right)$ Confidence intervals calculated with 2,000 bootstraps with 597 replacement and sample size of $50 \%$ of the diseases (150/299). $\left({ }^{* *}\right)$ Precision was 598 calculated based on the top 10 polyphenols after their ranking based on the distance $599\left(d_{c}\right)$ of their targets to the disease proteins and considering only predictions with Z-score $\left.600<-0.5 .{ }^{* * *}\right)$ Concentrations of polyphenols in blood were retrieved from the Human 601 Metabolome Database (HMDB)

\begin{tabular}{|c|c|c|c|c|c|c|}
\hline Polyphenol & AUC & AUC Cl* & Precision ${ }^{\star *}$ & $\begin{array}{c}\text { Concentration in } \\
\text { Blood }^{\star \star *}\end{array}$ & $\begin{array}{l}\text { N Mapped } \\
\text { Targets }\end{array}$ & $\begin{array}{l}\text { LCC } \\
\text { Size }\end{array}$ \\
\hline Coumarin & 0.93 & {$[0.86-0.98]$} & 0.6 & & 7 & 1 \\
\hline Piceatannol & 0.86 & {$[0.77-0.94]$} & 0.6 & & 39 & 23 \\
\hline Genistein & 0.82 & {$[0.75-0.89]$} & 0.7 & {$[0.006-0.525 u M]$} & 18 & 6 \\
\hline Ellagic acid & 0.79 & {$[0.63-0.92]$} & 0.6 & & 42 & 19 \\
\hline (-)-epigallocatechin 3-O-gallate & 0.78 & {$[0.70-0.86]$} & 0.8 & & 51 & 17 \\
\hline Isoliquiritigenin & 0.75 & {$[0.77-0.94]$} & 0.6 & & 10 & 8 \\
\hline Resveratrol & 0.75 & {$[0.66-0.82]$} & 1 & & 63 & 25 \\
\hline Pterostilbene & 0.73 & {$[0.61-0.84]$} & 0.6 & & 5 & 2 \\
\hline Quercetin & 0.73 & {$[0.64-0.81]$} & 1 & {$[0.022-0.080 \mathrm{uM}]$} & 216 & 140 \\
\hline (-)-epicatechin & 0.65 & {$[0.49-0.80]$} & 0.8 & 0.625 uM & 11 & 3 \\
\hline
\end{tabular}

602

603 
a

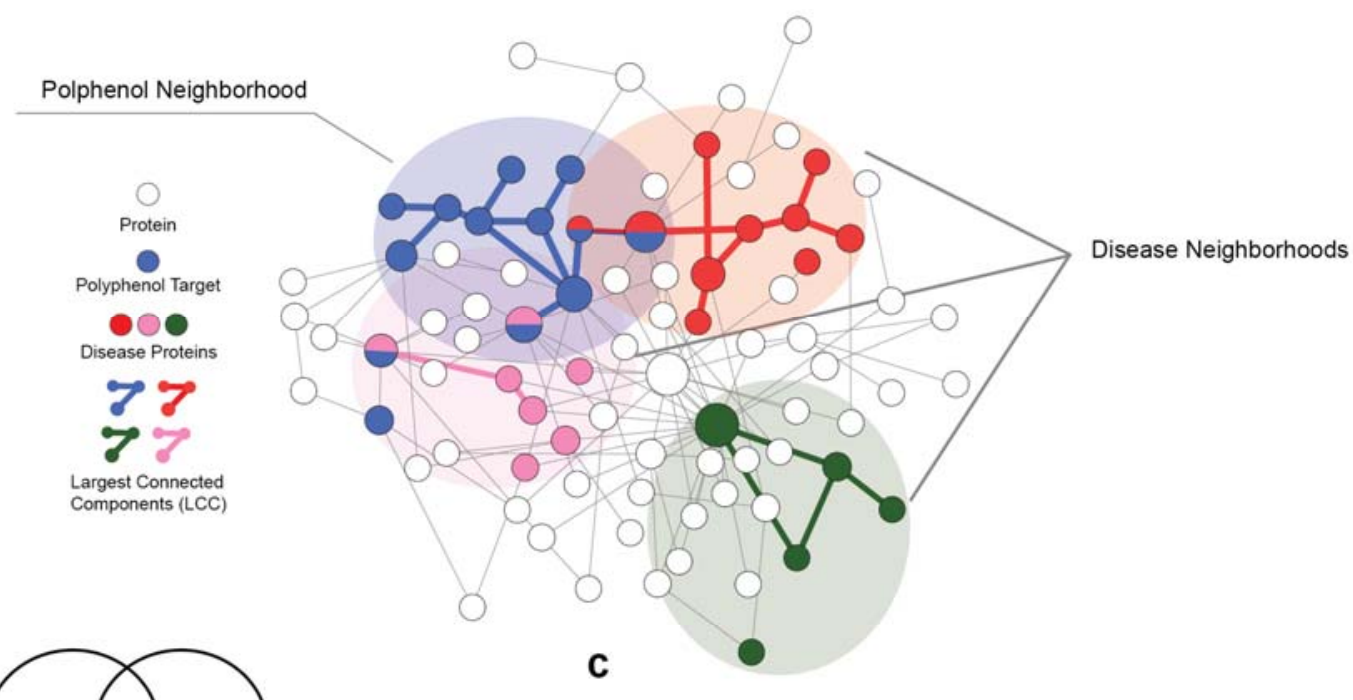

b
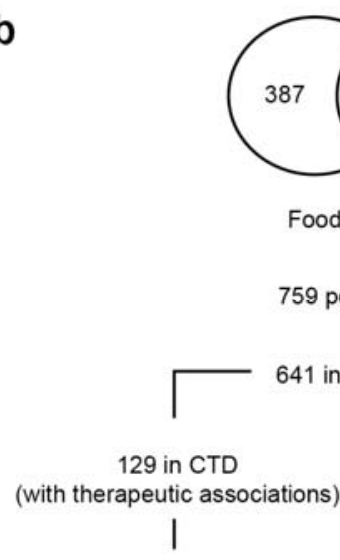

65 in STITCH

(experimental evidence

of protein interactions)

d

604

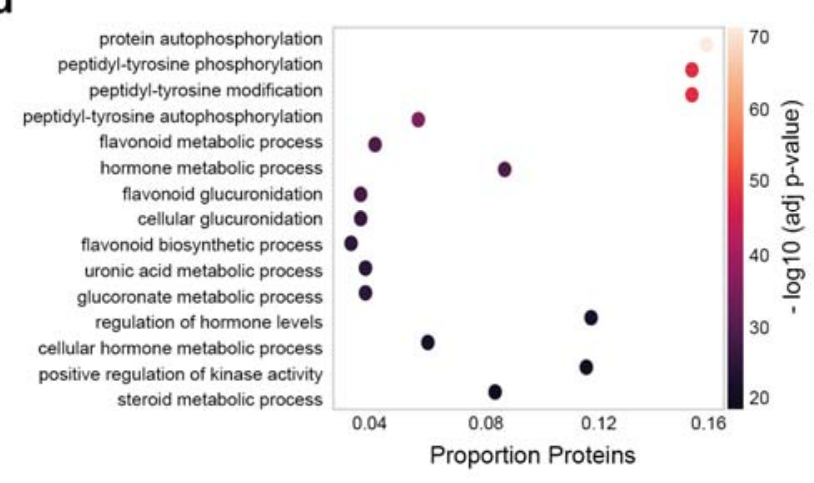

512 not in CTD

(no therapeutic associations)$$
\text { s) }
$$

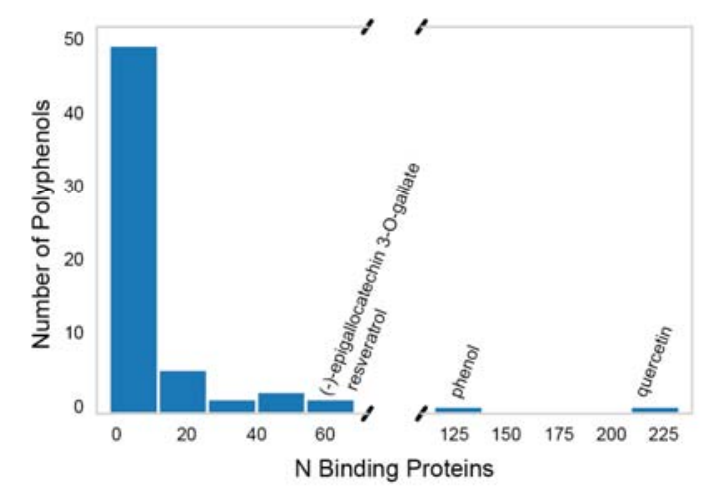

e

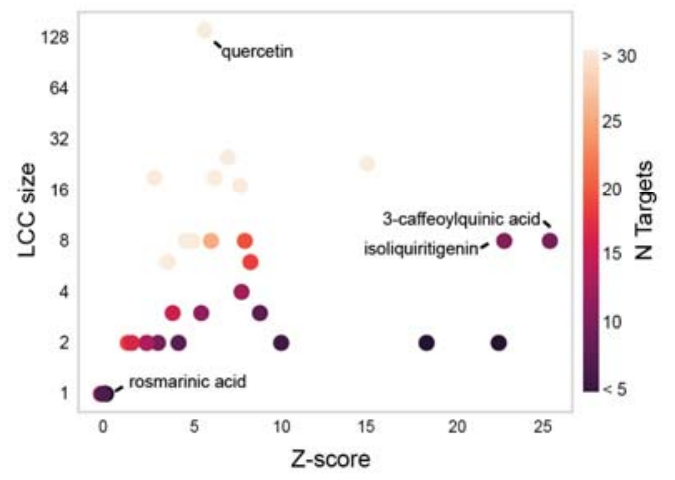

605

606

607

608

609

610

611

612

Figure 1 - Properties of Polyphenol Protein Targets. (A) Schematic representation of the human interactome, highlighting regions where polyphenol targets and disease proteins are localized. (B) Diagram showing the selection criteria of the polyphenols evaluated in this study. (C) Distribution of the number of polyphenol protein targets mapped to the human interactome. (D) Top $(n=15)$ enriched GO terms (Biological Process) among all polyphenol protein targets. The X-axis shows the proportion of targets mapped to each pathway. (E) Size of the Largest Connected Component (LCC) formed by the targets of each polyphenol in the interactome and the corresponding significance (z-score). 
bioRxiv preprint doi: https://doi.org/10.1101/2020.08 27 270173; this version posted February 8, 2021. The copyright holder for this preprint (which was not certified by peer review) is the author/funder, who has granted bioRxiv a license to display the preprint in perpetuity. It is made available under aCC-BY-NC-ND 4.0 International license.

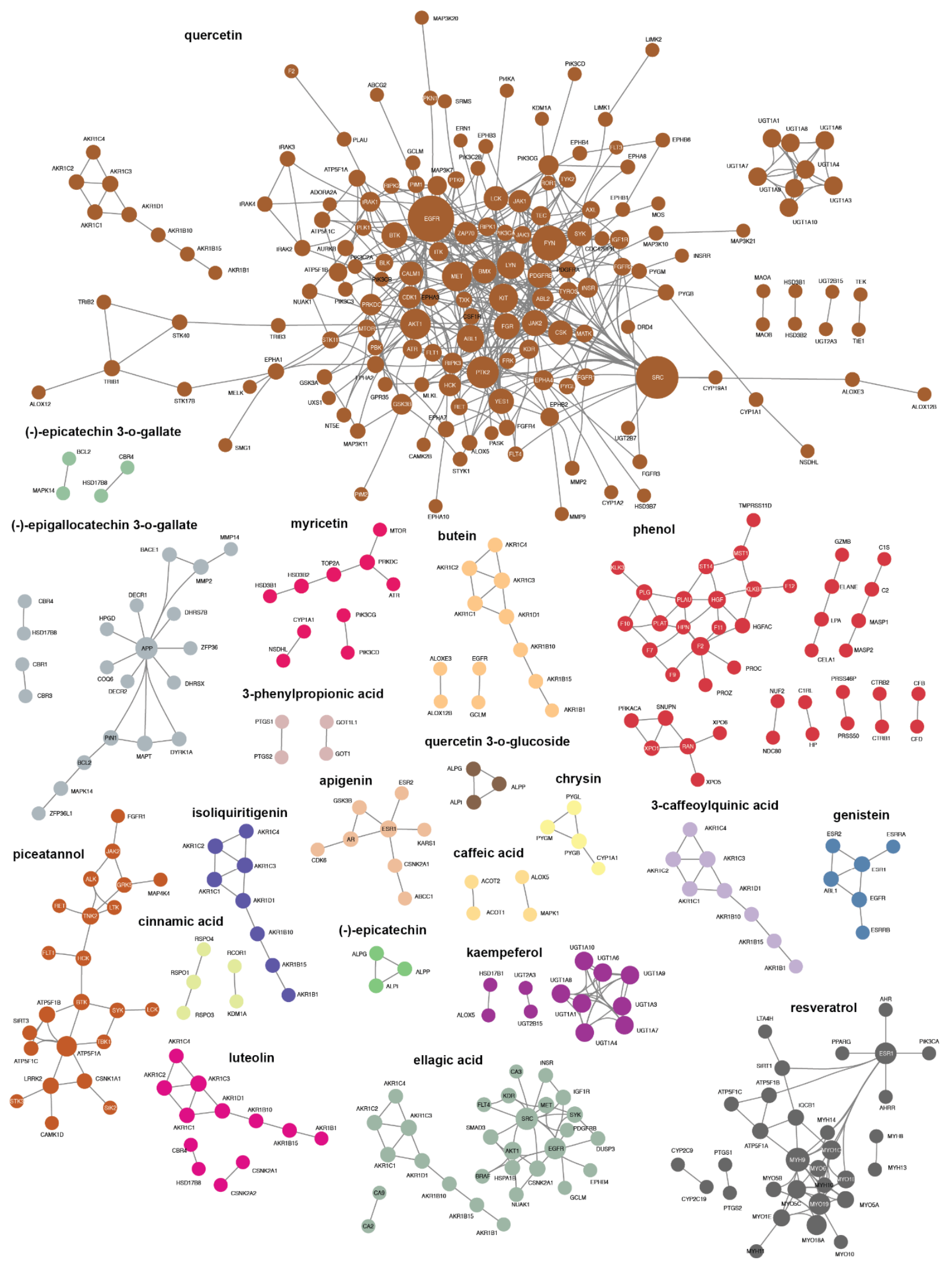

613 
bioRxiv preprint doi: https://doi.org/10.1101/2020.08.27.270173; this version posted February 8, 2021. The copyright holder for this preprint (which was not certified by peer review) is the author/funder, who has granted bioRxiv a license to display the preprint in perpetuity. It is made available under aCC-BY-NC-ND 4.0 International license.

614

615

616

617

618

619

Figure 2 - Protein-Protein Interactions of Polyphenol Targets. The 23 polyphenols whose targets form connected components in the interactome and their respective subgraphs. For example, piceatannol targets form a unique connected component of 23 proteins, while quercetin targets form multiple connected components, the largest with 140 proteins. Polyphenol targets that are not connected to any other target are not shown in the figure. Colors distinguish connected component of different polyphenols.

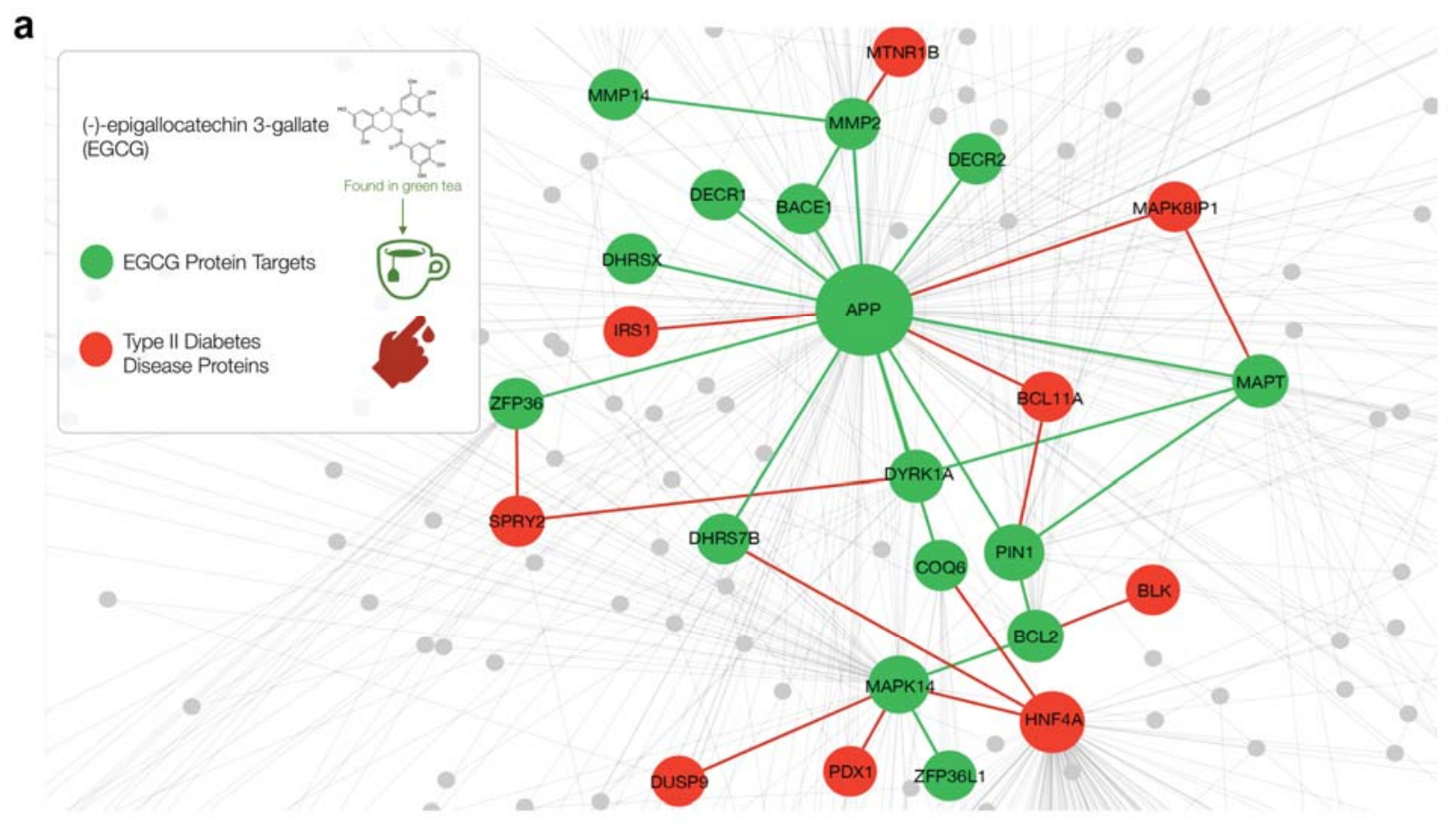

b

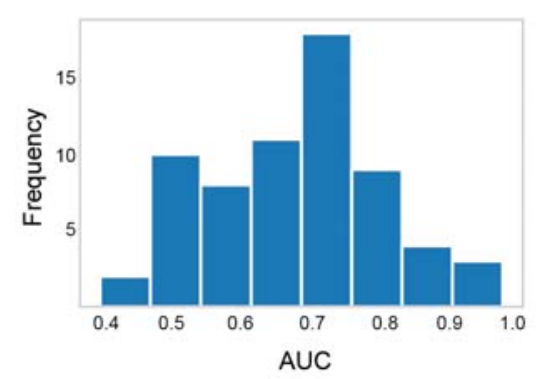

C

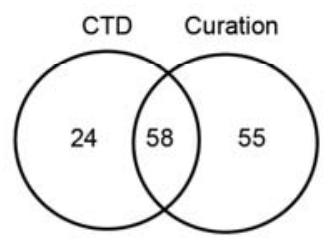

d

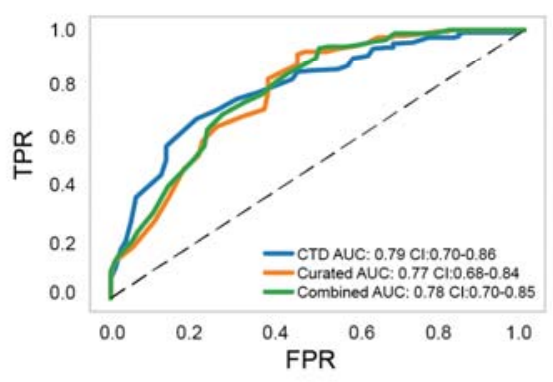

620

621

622

623

624

625

626

627

628
Figure 3 - Proximity Between Polyphenol Targets and Disease Proteins is Predictive of Therapeutic Effects of the Polyphenol. (a) Interactome neighborhood showing the EGCG protein targets and their interactions with type 2 diabetes (T2D)-associated proteins. (b) Distribution of AUC values considering the predictions of therapeutic effects for 65 polyphenols. (c) Comparison of the ECGCdisease associations considering the CTD database and the in-house database derived from the manual curation of the literature. (d) Comparison of the prediction performance when considering known EGCGdisease associations from the CTD, in-house manually curated database, or combined datasets. 


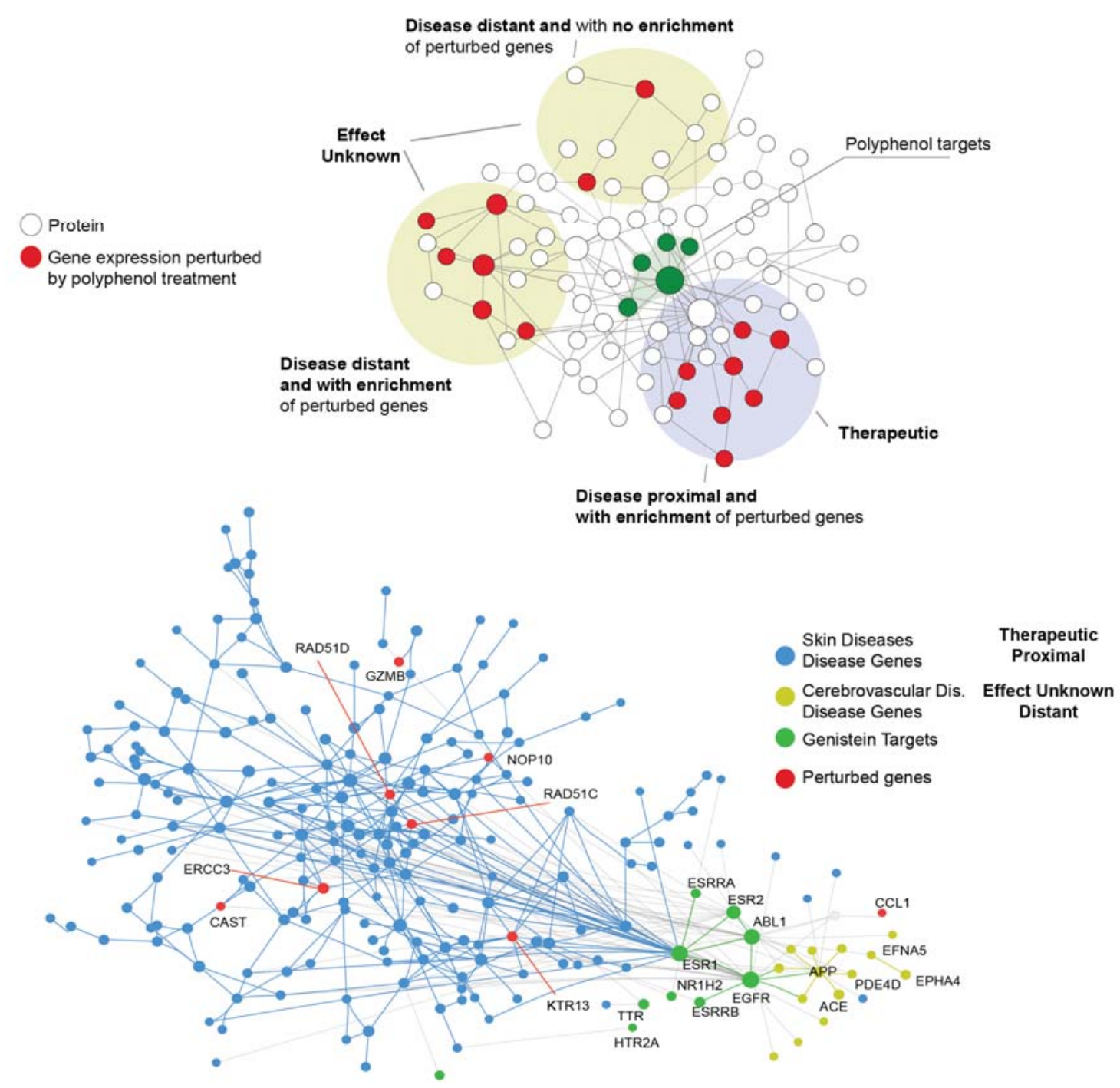

b

Gene expression perturbed by polyphenol treatment
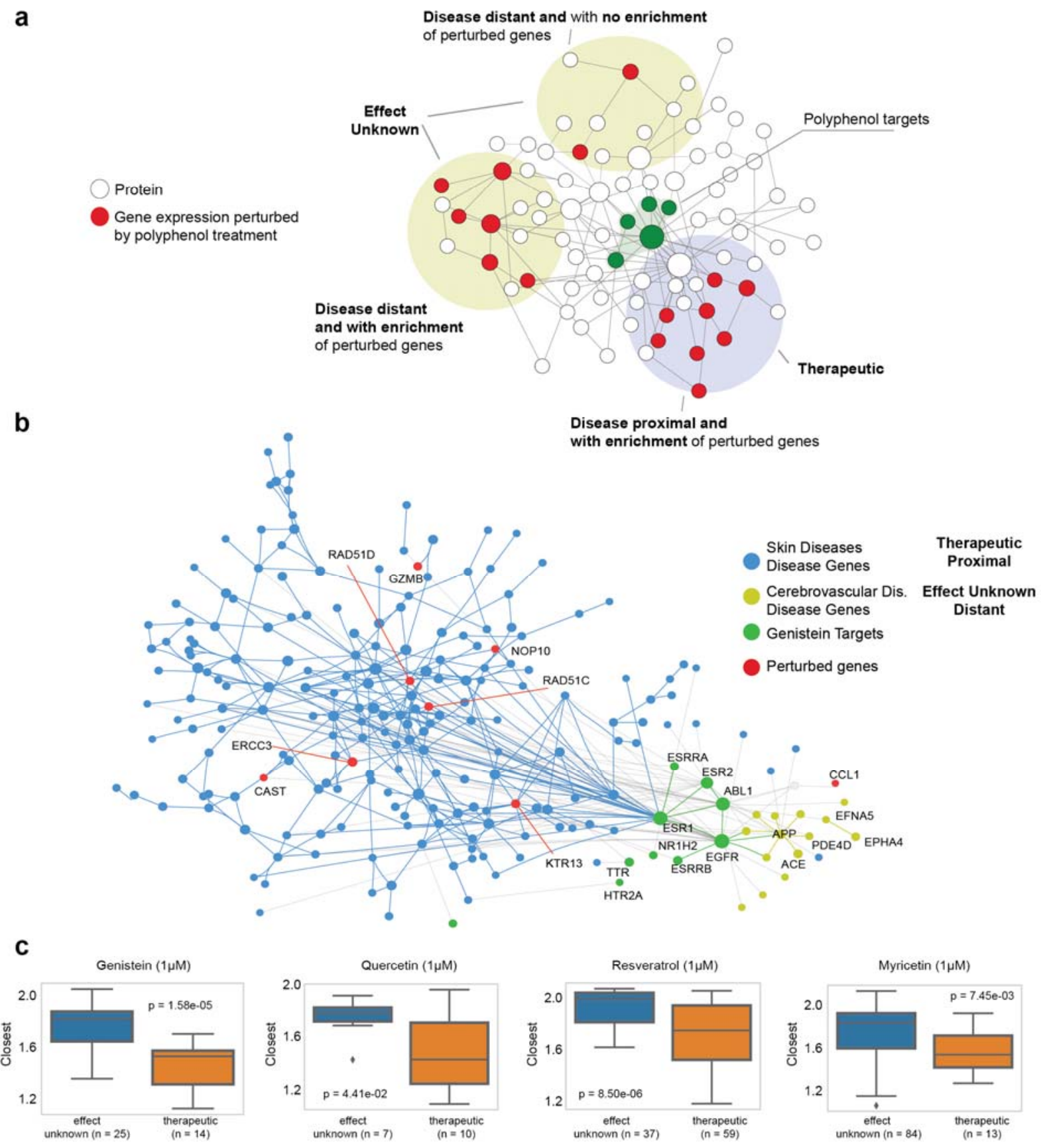

Figure 4 - Relationships among Gene Expression Perturbation, Network Proximity, and Therapeutic Effects of Polyphenols on Diseases. (a) Schematic representation of the relationship between the extent to which a polyphenol perturbs disease genes expression, its proximity to the disease genes, and its therapeutic effects. (b) Interactome neighborhood showing the modules of skin diseases (SD), genistein, and cerebrovascular disorders (CD). The SD module has 10 proteins with high perturbation scores (>2) in the treatment of the MCF7 cell line with $1 \mu \mathrm{M}$ of genistein. Genes associated to SD are significantly enriched among the most differentially expressed genes, and the maximum perturbation score among disease genes is higher in SD than CD. (c) Among the diseases in which genes are enriched with highly perturbed genes, those with therapeutic associations show smaller network distances to the polyphenol targets than those without. The same trend is observed in treatments of the polyphenols quercetin, resveratrol, and myricetin. 
a

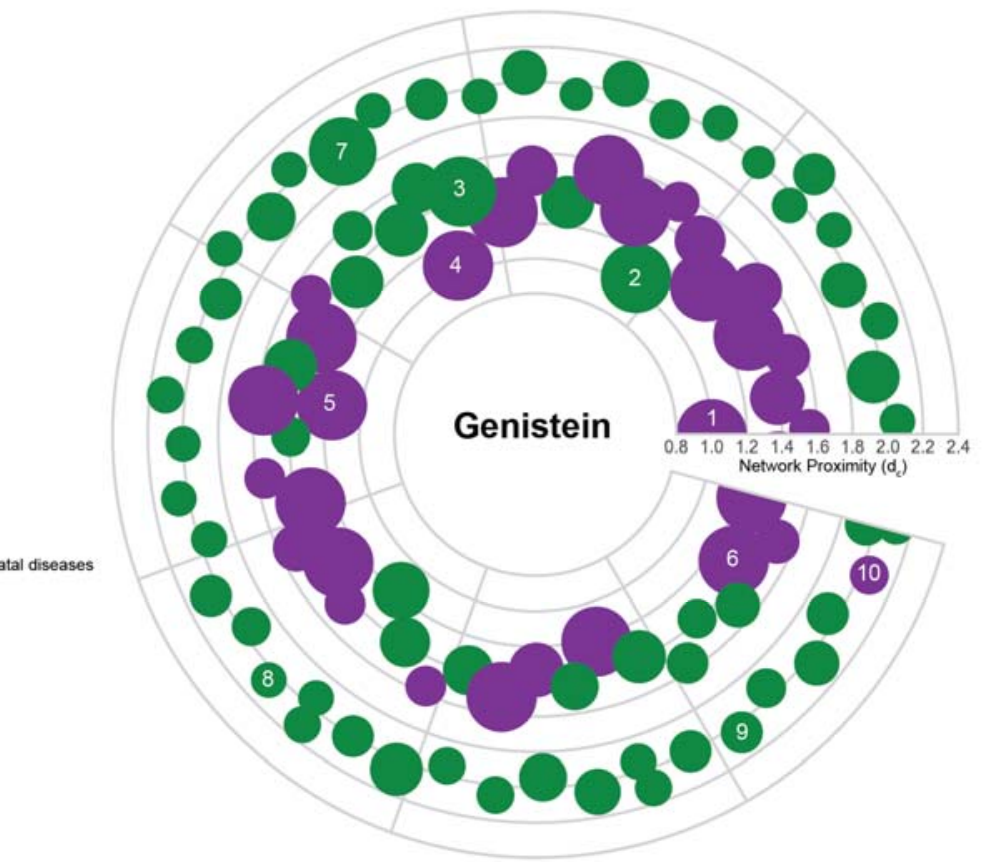

b

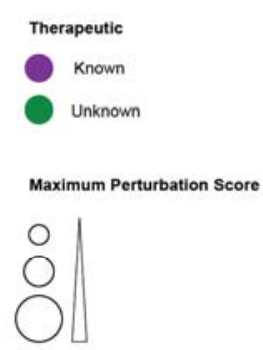

Disease Labels

1 - nervous systern diseases

2 - genetic diseases, inborn

3- metabolism, inborn errors

4 - congenital, hereditary, and neonatal diseases

5 - neoplasms

6 - colonic diseases

7 - macular degeneration

8 - spondylitis, ankylosing

9 - dyssomnias

10 - osteoporosis
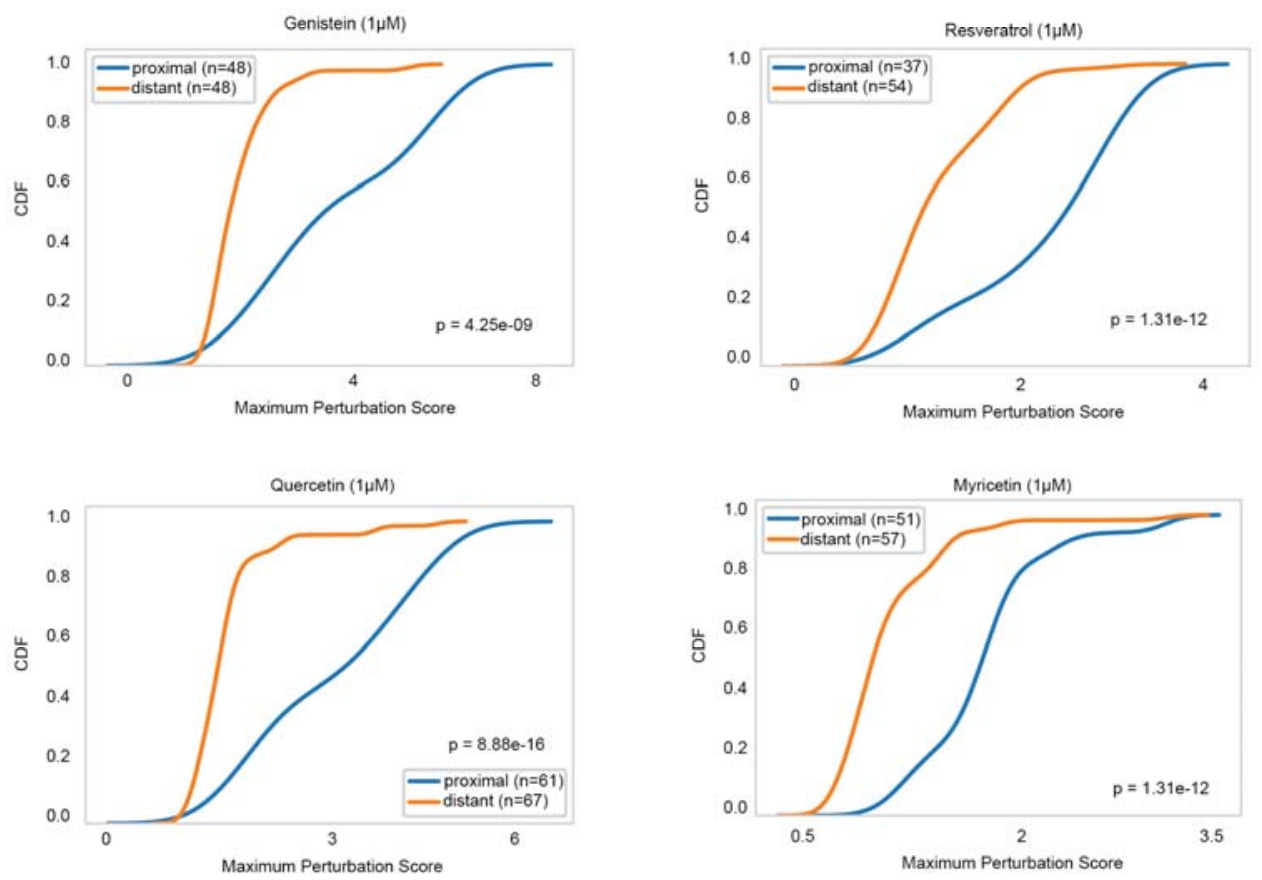

Figure 5 - Diseases Proximal to Polyphenol Targets Have Higher Gene Expression Perturbation

Profiles. (a) Proximal and distal diseases in relation to genistein targets. Each node represents a disease and the node size is proportional to the perturbation score after treatment with genistein $(1 \mu \mathrm{M}, 6$ hours). Distance from the origin represents the network proximity $\left(d_{c}\right)$ to genistein targets. Purple nodes represent diseases in which the therapeutic association was previously known. (c) Cumulative distribution of the maximum perturbation scores of genes from diseases that are distal or proximal to polyphenol targets considering different polyphenols ( $1 \mu \mathrm{M}, 6$ hours): genistein, quercetin, resveratrol, and myricetin. Statistical significance was evaluated with the Kolmogorov-Smirnov test. 


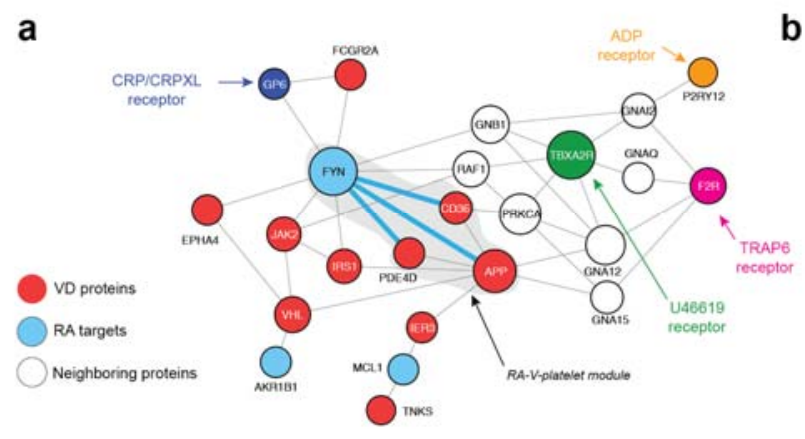

C
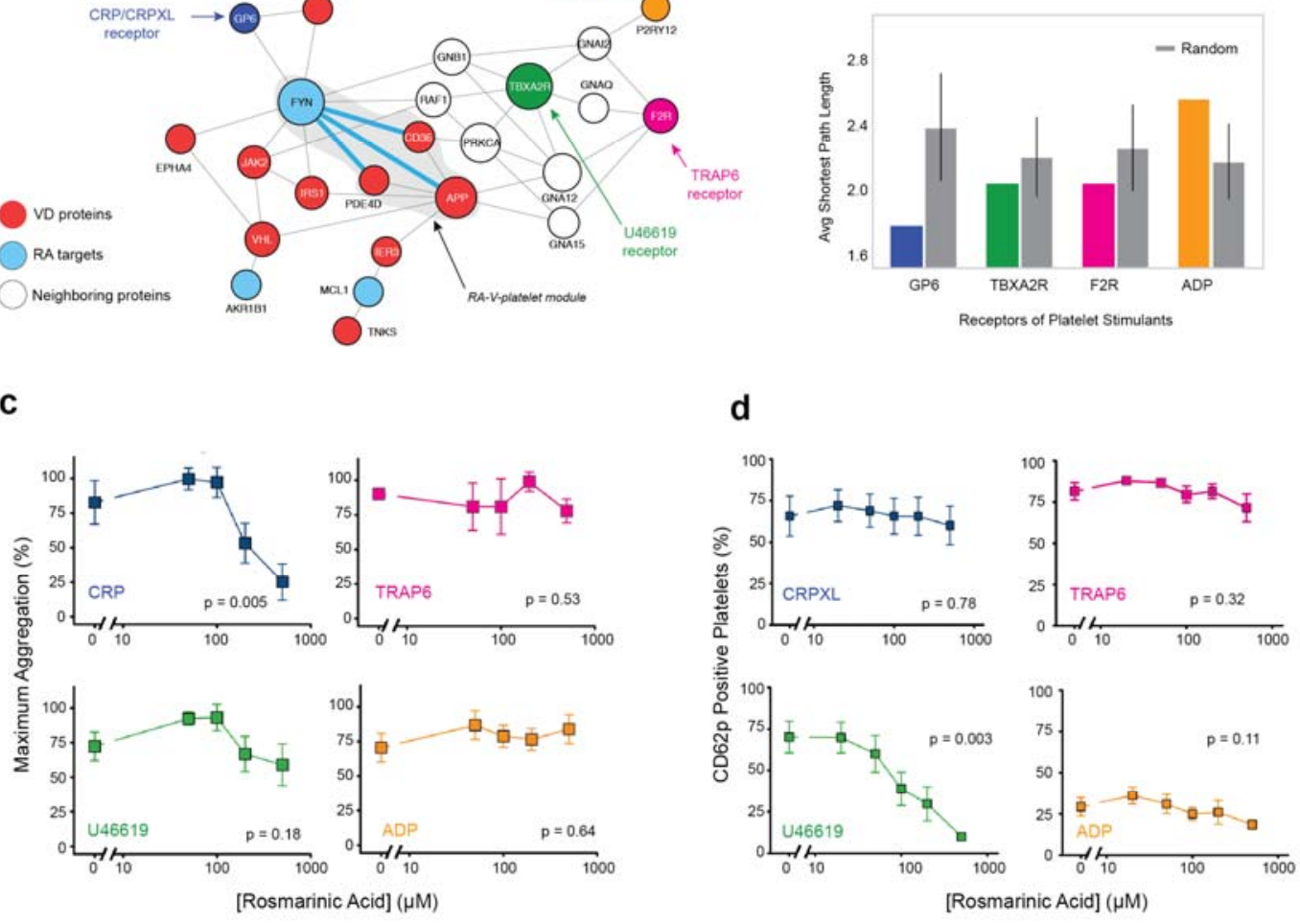

f
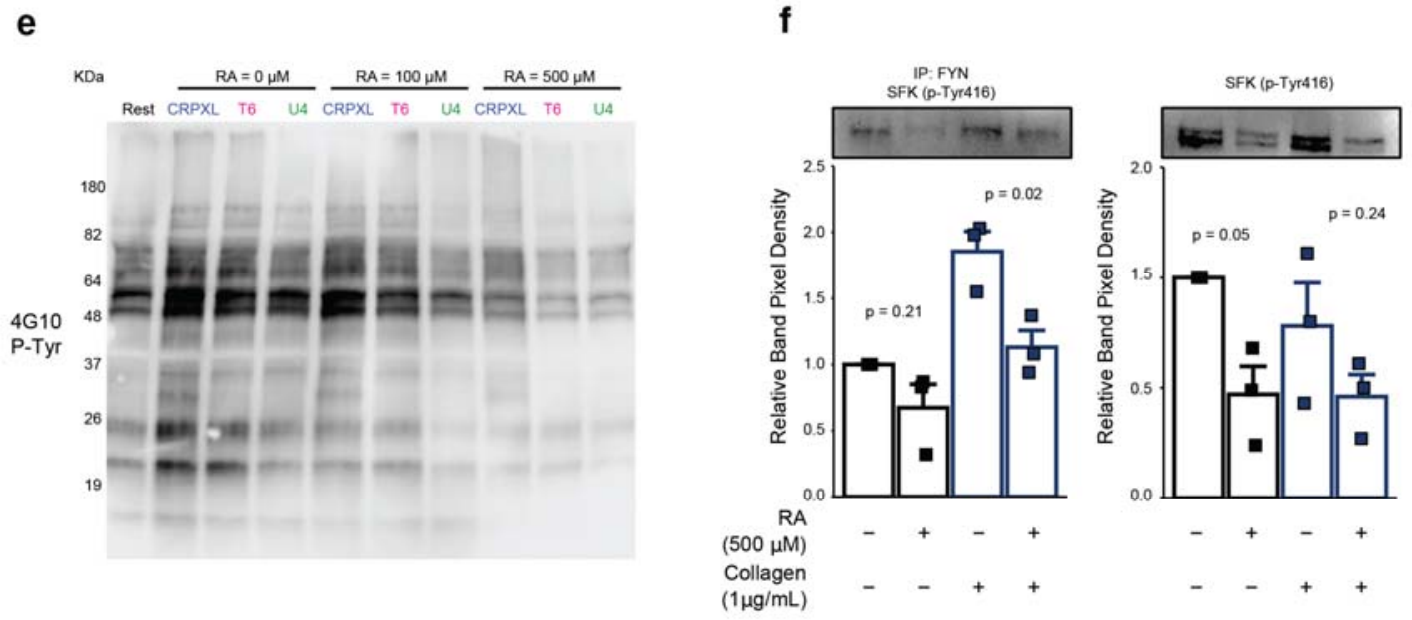

Figure 6 - Rosmarinic Acid Modulates Platelet Function. (a) Interactome neighborhood showing rosmarinic acid (RA) targets and the RA-V-platelet module - the connected component formed by the RA target FYN and the $V$ proteins associated with platelet function PDE4D, CD36, and APP - and the receptor for platelet agonists used in our experiments (collagen/CRPXL, TRAP6, U46619, and ADP). (b) Average shortest path length from each platelet agonist receptor and the RA-V-platelet module formed by the proteins FYN, PDE4D, CD36, APP. Bars represent standard deviation of that same measure over 1000 iterations of random selection of nodes in a degree preserving fashion. c-e) Platelet-rich plasma (PRP) or washed platelets were pre-treated with RA for 1 hour before stimulation with either collagen (1 $\mu \mathrm{g} / \mathrm{mL}$ ), collagen-related peptide (CRP-XL, $1 \mu \mathrm{g} / \mathrm{mL}$ ), thrombin receptor activator peptide-6 (TRAP-6, 20 $\mu \mathrm{M}), \mathrm{U} 46619(1 \mu \mathrm{M})$, or ADP $(10 \mu \mathrm{M})$. Platelets were assessed for either (c) aggregation, (d) alpha 
bioRxiv preprint doi: https://doi org/10.1101/2020.08.27.270173; this version posted February 8, 2021. The copyright holder for this preprint (which was not certified by peer review) is the author/funder, who has granted bioRxiv a license to display the preprint in perpetuity. It is made available under aCC-BY-NC-ND 4.0 International license.

663 granule secretion. Platelet lysates were also probed for either (e) non-specific tyrosine phosphorylation 664 (p-Tyr) of the whole cell lysate, or (d) site-specific phosphorylation of src family kinases (SFKs) and FYN 665 at residue 416. $n=3-6$ separate blood donations, mean +/- SEM. $p$-values in (c) and (d) were determined 666 by Kruskal-Wallis test and by unpaired t.tests in (f).

667 
670 1. Khera, A. V. et al. Genetic Risk, Adherence to a Healthy Lifestyle, and Coronary Disease. N. Engl. J. Med. 375, 2349-2358 (2016).

672 2. Arts, I. C. W. \& Hollman, P. C. H. Polyphenols and disease risk in epidemiologic studies. Am. J. Clin. Nutr. 81, 317S-325S (2005).

3. Wang, X., Ouyang, Y. Y., Liu, J. \& Zhao, G. Flavonoid intake and risk of CVD: A systematic review and meta-analysis of prospective cohort studies. Br. J. Nutr. 111, 1-11 (2014).

4. Neveu, V. et al. Phenol-Explorer: an online comprehensive database on polyphenol contents in foods. Database 2010, bap024-bap024 (2010).

6. Zhang, H. \& Tsao, R. Dietary polyphenols, oxidative stress and antioxidant and

5. Pérez-Jiménez, J., Neveu, V., Vos, F. \& Scalbert, A. Systematic analysis of the content of 502 Polyphenols in 452 foods and beverages: An application of the

8. Lacroix, S. et al. A computationally driven analysis of the polyphenol-protein interactome. Sci. Rep. 8, 2232 (2018).

9. Hanhineva, K. et al. Impact of dietary polyphenols on carbohydrate metabolism. Int. J. Mol. Sci. 11, 1365-402 (2010).

10. Hervert-Hernández, D. \& Goñi, I. Dietary polyphenols and human gut microbiota: A review. Food Rev. Int. 27, 154-169 (2011).

11. Zhang, S. et al. Dietary pomegranate extract and inulin affect gut microbiome differentially in mice fed an obesogenic diet. Anaerobe 48, 184-193 (2017).

12. Thazhath, S. S. et al. Administration of resveratrol for 5 wk has no effect on glucagon-like peptide 1 secretion, gastric emptying, or glycemic control in type 2 diabetes: a randomized controlled trial. Am. J. Clin. Nutr. 103, 66-70 (2016).
13. Bhatt, J. K., Thomas, S. \& Nanjan, M. J. Resveratrol supplementation improves 
glycemic control in type 2 diabetes mellitus. Nutr. Res. 32, 537-41 (2012).

14. Sharma, A. et al. A disease module in the interactome explains disease Hum. Mol. Genet. 24, 3005-3020 (2014).

15. Menche, J. et al. Disease networks. Uncovering disease-disease relationships through the incomplete interactome. Science 347, 1257601 (2015).

16. Guney, E., Menche, J., Vidal, M. \& Barabási, A.-L. Network-based in silico drug efficacy screening. Nat. Commun. 7, 10331 (2016).

17. Cheng, F. et al. Network-based approach to prediction and population-based validation of in silico drug repurposing. Nat. Commun. 9, 1-12 (2018).

18. Kovács, I. A. et al. Network-based prediction of protein interactions. Nat.

Commun. 10, 1240 (2019).

19. Sarkar, F. H., Li, Y., Wang, Z. \& Kong, D. Cellular signaling perturbation by natural products. Cell. Signal. 21, 1541-7 (2009).

20. Iso, H. et al. The relationship between green tea and total caffeine intake and risk for self-reported type 2 diabetes among Japanese adults. Ann. Intern. Med. 144, 554-62 (2006).

21. Song, Y., Manson, J. E., Buring, J. E., Sesso, H. D. \& Liu, S. Associations of dietary flavonoids with risk of type 2 diabetes, and markers of insulin resistance and systemic inflammation in women: a prospective study and cross-sectional analysis. J. Am. Coll. Nutr. 24, 376-84 (2005).

22. Keske, M. A. et al. Vascular and metabolic actions of the green tea polyphenol epigallocatechin gallate. Curr. Med. Chem. 22, 59-69 (2015).

23. Wolfram, S. et al. Epigallocatechin gallate supplementation alleviates diabetes in

724 24. Muthu, R., Selvaraj, N. \& Vaiyapuri, M. Anti-inflammatory and proapoptotic effects of umbelliferone in colon carcinogenesis. Hum. Exp. Toxicol. 35, 1041-54 (2016).

726 25. Muthu, R. \& Vaiyapuri, M. Synergistic and individual effects of umbelliferone with 5-fluorouracil on tumor markers and antioxidant status of rat treated with 1,2dimethylhydrazine. Biomed. Aging Pathol. 3, 219-227 (2013).

26. Subramanian, A. et al. A Next Generation Connectivity Map: L1000 Platform and 
the First 1,000,000 Profiles. Cell 171, 1437-1452.e17 (2017).

27. Grover, S. P., Bergmeier, W. \& Mackman, N. Platelet Signaling Pathways and New Inhibitors. Arterioscler. Thromb. Vasc. Biol. 38, e28-e35 (2018).

28. Moco, S., Martin, F. P. J. \& Rezzi, S. Metabolomics view on gut microbiome modulation by polyphenol-rich foods. J. Proteome Res. 11, 4781-4790 (2012).

29. van Duynhoven, J. et al. Metabolic fate of polyphenols in the human superorganism. Proc. Natl. Acad. Sci. 108, 4531-4538 (2011).

30. Ottaviani, J. I., Heiss, C., Spencer, J. P. E., Kelm, M. \& Schroeter, H. Recommending flavanols and procyanidins for cardiovascular health: Revisited. Mol. Aspects Med. 61, 63-75 (2018).

31. Stalmach, A., Troufflard, S., Serafini, M. \& Crozier, A. Absorption, metabolism and excretion of Choladi green tea flavan-3-ols by humans. Mol. Nutr. Food Res. (2009) doi:10.1002/mnfr.200800169.

32. Meng, X. et al. Identification and characterization of methylated and ring-fission metabolites of tea catechins formed in humans, mice, and rats. Chem. Res. Toxicol. (2002) doi:10.1021/tx010184a.

33. Perez-Vizcaino, F., Duarte, J. \& Santos-Buelga, C. The flavonoid paradox: Conjugation and deconjugation as key steps for the biological activity of flavonoids. J. Sci. Food Agric. 92, 1822-1825 (2012).

34. Shimoi, K. \& Nakayama, T. Glucuronidase deconjugation in inflammation. Methods Enzymol. 400, 263-272 (2005).

35. Kaneko, A. et al. Glucuronides of phytoestrogen flavonoid enhance macrophage function via conversion to aglycones by $\beta$-glucuronidase in macrophages. Immun. Inflamm. Dis. 5, 265-279 (2017).

36. Cheng, F., Kovács, I. A. \& Barabási, A.-L. Network-based prediction of drug

759 38. Lamb, J. et al. The Connectivity Map: using gene-expression signatures to combinations. Nat. Commun. 10, 1197 (2019).

37. Smalley, J. L., Gant, T. W. \& Zhang, S.-D. Application of connectivity mapping in predictive toxicology based on gene-expression similarity. Toxicology 268, 143146 (2010). connect small molecules, genes, and disease. Science 313, 1929-35 (2006). 
39. Amanzadeh, E. et al. Quercetin conjugated with superparamagnetic iron oxide nanoparticles improves learning and memory better than free quercetin via interacting with proteins involved in LTP. Sci. Rep. 9, 1-19 (2019).

40. Shaikh, J., Ankola, D. D. D., Beniwal, V., Singh, D. D. \& Kumar, M. N. V. R. Nanoparticle encapsulation improves oral bioavailability of curcumin by at least 9fold when compared to curcumin administered with piperine as absorption enhancer. Eur. J. Pharm. Sci. 37, 223-30 (2009).

41. Chao, E. C. \& Henry, R. R. SGLT2 inhibition-A novel strategy for diabetes treatment. Nat. Rev. Drug Discov. 9, 551-559 (2010).

42. Caldera, M. et al. Mapping the perturbome network of cellular perturbations. Nat. Commun. 10, (2019).

43. Jensen, K., Ni, Y., Panagiotou, G. \& Kouskoumvekaki, I. Developing a Molecular Roadmap of Drug-Food Interactions. PLoS Comput. Biol. 11, 1-15 (2015).

44. Rolland, T. et al. A proteome-scale map of the human interactome network. Cell 159, 1212-1226 (2014).

45. Cheng, F., Jia, P., Wang, Q. \& Zhao, Z. Quantitative network mapping of the human kinome interactome reveals new clues for rational kinase inhibitor discovery and individualized cancer therapy. Oncotarget 5, 3697-710 (2014).

47. Hornbeck, P. V et al. PhosphoSitePlus, 2014: mutations, PTMs and recalibrations. Nucleic Acids Res. 43, D512-20 (2015).

48. $\mathrm{Li}, \mathrm{T}$. et al. A scored human protein-protein interaction network to catalyze genomic interpretation. Nat. Methods 14, 61-64 (2016).

49. Chatr-Aryamontri, A. et al. The BioGRID interaction database: 2017 update. Nucleic Acids Res. 45, D369-D379 (2017).

788 50. Cowley, M. J. et al. PINA v2.0: mining interactome modules. Nucleic Acids Res. 40, D862-5 (2012).

790 51. Peri, S. et al. Human protein reference database as a discovery resource for proteomics. Nucleic Acids Res. 32, D497-501 (2004). 
792 52. Orchard, S. et al. The MIntAct project--IntAct as a common curation platform for 11 molecular interaction databases. Nucleic Acids Res. 42, D358-63 (2014).

794 53. Breuer, K. et al. InnateDB: systems biology of innate immunity and beyond-recent updates and continuing curation. Nucleic Acids Res. 41, D1228-33 (2013).

54. Meyer, M. J., Das, J., Wang, X. \& Yu, H. INstruct: a database of high-quality 3D structurally resolved protein interactome networks. Bioinformatics 29, 1577-9 (2013).

55. Mosca, R., Céol, A. \& Aloy, P. Interactome3D: adding structural details to protein networks. Nat. Methods 10, 47-53 (2013).

56. Meyer, M. J. et al. Interactome INSIDER: a structural interactome browser for genomic studies. Nat. Methods 15, 107-114 (2018).

57. Fazekas, D. et al. SignaLink 2 - a signaling pathway resource with multi-layered regulatory networks. BMC Syst. Biol. 7, 7 (2013).

58. Huttlin, E. L. et al. Architecture of the human interactome defines protein communities and disease networks. Nature 545, 505-509 (2017).

59. Davis, A. P. et al. The Comparative Toxicogenomics Database: update 2019. Nucleic Acids Res. 47, D948-D954 (2019).

60. Szklarczyk, D. et al. STRING v10: protein-protein interaction networks, integrated over the tree of life. Nucleic Acids Res. 43, D447-52 (2015).

61. Subramanian, A. et al. Gene set enrichment analysis: a knowledge-based approach for interpreting genome-wide expression profiles. Proc. Natl. Acad. Sci. U. S. A. 102, 15545-50 (2005).

62. Roweth, H. G. et al. Two novel, putative mechanisms of action for citalopraminduced platelet inhibition. Sci. Rep. 8, 1-14 (2018).

63. Roweth, H. G. et al. Citalopram inhibits platelet function independently of SERTmediated 5-HT transport. Sci. Rep. 8, 1-14 (2018).

64. Nath, S., Bachani, M., Harshavardhana, D. \& Steiner, J. P. Catechins protect neurons against mitochondrial toxins and HIV proteins via activation of the BDNF

821 65. Park, K.-S. et al. (-)-Epigallocatethin-3-O-gallate counteracts caffeine-induced 
824 66. Ramesh, E., Geraldine, P. \& Thomas, P. A. Regulatory effect of epigallocatechin gallate on the expression of C-reactive protein and other inflammatory markers in an experimental model of atherosclerosis. Chem. Biol. Interact. 183, 125-32 (2010).

67. Han, S. G., Han, S.-S., Toborek, M. \& Hennig, B. EGCG protects endothelial cells against PCB 126-induced inflammation through inhibition of AhR and induction of Nrf2-regulated genes. Toxicol. Appl. Pharmacol. 261, 181-8 (2012).

68. Sheng, R., Gu, Z.-L. \& Xie, M.-L. Epigallocatechin gallate, the major component of polyphenols in green tea, inhibits telomere attrition mediated cardiomyocyte apoptosis in cardiac hypertrophy. Int. J. Cardiol. 162, 199-209 (2013).

69. Devika, P. T. \& Stanely Mainzen Prince, P. (-)-Epigallocatechin gallate protects the mitochondria against the deleterious effects of lipids, calcium and adenosine triphosphate in isoproterenol induced myocardial infarcted male Wistar rats. J. Appl. Toxicol. 28, 938-44 (2008).

70. Yi, Q.-Y. et al. Chronic infusion of epigallocatechin-3-O-gallate into the

71. Devika, P. T. \& Prince, P. S. M. Preventive effect of (-)epigallocatechin-gallate (EGCG) on lysosomal enzymes in heart and subcellular fractions in isoproterenolinduced myocardial infarcted Wistar rats. Chem. Biol. Interact. 172, 245-52 (2008).

72. Hushmendy, S. et al. Select phytochemicals suppress human T-lymphocytes and mouse splenocytes suggesting their use in autoimmunity and transplantation.

Res. 29, 568-78 (2009).

849 73. Shen, K. et al. Epigallocatechin 3-Gallate Ameliorates Bile Duct Ligation Induced Liver Injury in Mice by Modulation of Mitochondrial Oxidative Stress and

852 74. ZHEN, M. et al. Green tea polyphenol epigallocatechin-3-gallate inhibits oxidative 
$854 \quad$ J. Nutr. Biochem. 18, 795-805 (2007).

855 75. Yasuda, Y. et al. (-)-Epigallocatechin gallate prevents carbon tetrachloride-

856 induced rat hepatic fibrosis by inhibiting the expression of the PDGFR $\beta$ and IGF-

857 1R. Chem. Biol. Interact. 182, 159-164 (2009).

858 76. Cao, W. et al. iTRAQ-based proteomic analysis of combination therapy with

859 taurine, epigallocatechin gallate, and genistein on carbon tetrachloride-induced

$860 \quad$ liver fibrosis in rats. Toxicol. Lett. 232, 233-245 (2015).

861 77. Kitamura, M. et al. Epigallocatechin gallate suppresses peritoneal fibrosis in mice.

862 Chem. Biol. Interact. 195, 95-104 (2012).

863 78. Sakla, M. S. \& Lorson, C. L. Induction of full-length survival motor neuron by

864 polyphenol botanical compounds. Hum. Genet. 122, 635-643 (2008).

865 79. Shimizu, M. et al. (-)-Epigallocatechin gallate inhibits growth and activation of the 866 VEGF/VEGFR axis in human colorectal cancer cells. Chem. Biol. Interact. 185, $867 \quad 247-252(2010)$. 